\title{
Anti-Osteoporosis Effects of the Eleutherococcus senticosus, Achyranthes japonica, and Atractylodes japonica Mixed Extract Fermented with Nuruk
}

\author{
So Young Eun ${ }^{1,+}+\mathbb{D}$, Yoon-Hee Cheon ${ }^{1,+}$, Gyeong Do Park ${ }^{1}$, Chong Hyuk Chung ${ }^{1,2}$, Chang Hoon Lee ${ }^{1,2}$, \\ Ju-Young Kim ${ }^{1, * \mathbb{D}}$ and Myeung Su Lee ${ }^{1,2, *}$ \\ 1 Musculoskeletal and Immune Disease Research Institute, School of Medicine, Wonkwang University, \\ 460 Iksandae-ro, Iksan 54538, Korea; eunsoyg@hanmail.net (S.Y.E.); hanleuni@naver.com (Y.-H.C.); \\ rudeh2508@naver.com (G.D.P.); taylorchung@hanmail.net (C.H.C.); lch110@wku.ac.kr (C.H.L.) \\ 2 Division of Rheumatology, Department of Internal Medicine, Wonkwang University Hospital, \\ 460 Iksandae-ro, Iksan 54538, Korea \\ * Correspondence: kimjy1014@gmail.com (J.-Y.K.); ckhlms@wku.ac.kr (M.S.L.); Tel.: +82-63-850-6088 (J.-Y.K.); \\ +82-63-859-2661 (M.S.L.) \\ + These authors contributed equally to this work.
}

check for

updates

Citation: Eun, S.Y.; Cheon, Y.-H.; Park, G.D.; Chung, C.H.; Lee, C.H.; Kim, J.-Y.; Lee, M.S. Anti-Osteoporosis Effects of the Eleutherococcus senticosus, Achyranthes japonica, and Atractylodes japonica Mixed Extract Fermented with Nuruk. Nutrients 2021, 13, 3904. https://doi.org/ 10.3390/nu13113904

Academic Editor: Andrea Fabbri

Received: 5 October 2021

Accepted: 28 October 2021

Published: 30 October 2021

Publisher's Note: MDPI stays neutral with regard to jurisdictional claims in published maps and institutional affiliations.

Copyright: (c) 2021 by the authors. Licensee MDPI, Basel, Switzerland. This article is an open access article distributed under the terms and conditions of the Creative Commons Attribution (CC BY) license (https:/ / creativecommons.org/licenses/by/ $4.0 /)$.

\begin{abstract}
Vigeo is a mixture of fermented extracts of Eleutherococcus senticosus Maxim (ESM), Achyranthes japonica (Miq.) Nakai (AJN), and Atractylodes japonica Koidzumi (AJK) manufactured using the traditional Korean nuruk fermentation method. Although the bioactive effects of ESM, AJN, and AJK have already been reported, the pharmacological effects of Vigeo have not been proven. Therefore, in this study, we investigated whether Vigeo had inhivitory effects on lipopolysaccharide (LPS)-induced inflammatory bone loss in vivo and receptor activator of nuclear factor-B ligand (RANKL)-induced osteoclastogenesis and the related mechanism in vitro. Vigeo administration conferred effective protection against bone loss induced by excessive inflammatory response and activity of osteoclasts in LPS-induced inflammatory osteoporosis mouse model. In addition, Vigeo significantly suppressed the formation of tartrate-resistant acid phosphatase-positive osteoclasts induced by RANKL and inhibited F-actin formation and bone resorbing activity without any cytotoxicity. Moreover, Vigeo significantly inhibited RANKL-induced phosphorylation of p38, ERK, JNK, IKB, and AKT and degradation of IkB. Additionally, Vigeo strongly inhibited the mRNA and protein expression of c-FOS and NFATc1 and subsequently attenuated the expression of osteoclast specific marker genes induced by RANKL. We demonstrated for the first time the anti-osteoporosis effect of Vigeo, suggesting that it could be a potential therapeutic candidate for the treatment of osteoclast-mediated inflammatory bone diseases.
\end{abstract}

Keywords: Vigeo; nuruk fermentation; osteoclasts; bone resorption; osteoporosis

\section{Introduction}

Osteoporosis is a common disease caused by problems with the bone remodeling process and is characterized by a reduction in bone mass and bone microstructural alterations [1]. Although the pathogenesis of bone remodeling is not completely understood, it is clear that an imbalance in bone remodeling, osteoporotic fractures, and vertebral and hip fractures are associated with morbidity and increased mortality [1].

In order to counteract the side effects of osteoporosis drugs currently in use, many researchers have tried to develop new treatments for bone metabolic disorders based on natural plant-derived compounds. The use of plants for treatment of several diseases has a long history [2,3]. Numerous plant extracts have been investigated for their antiosteoporosis activity, and some have been reported to have potent activity [4,5]. In addition, fermentation with plant products may further enhance the functional properties of medicinal plants or attenuate their toxicity and side effects [6,7]. In the fermentation process, 
complex substances are broken down into smaller molecules by microorganisms to generate stable products, which improves their pharmacological efficacy for disease prevention [8].

Vigeo is a fermented combined extract of Eleutherococcus senticosus Maxim (ESM), Achyranthes japonica (Miq.) Nakai (AJN), and Atractylodes japonica Koidzumi (AJK), using the traditional Korean nuruk fermentation method. ESM, also called Siberian ginseng, has been reported to protect the bone from damage as well as to exert an anti-osteonecrotic effect in vitro and in vivo [9]. Notably, treatment with ESM may potentially overcome the effects of inflammation in the bone [10], as ESM can reduce calcium and hydroxyproline excretion in glucocorticoid-induced osteoporotic model [11]. Moreover, a recent study demonstrated that ESM extract has an inhibitory effect on RANKL-induced osteoclast formation [12]. AJN is traditional medicine in Korea, Japan, and China, used as a remedy for pain control and improvement of functional disorders in patients with osteoarthritis [13]. AJN is a herbal medicine traditionally used for the treatment of obesity and related complications [14], reported to have anti-inflammatory [15], anti-arthritic [16], and antioxidant properties [17] and. AJK also exhibits a wide variety of bioactive properties, including anti-inflammatory [18], antioxidant [19], and anti-obesity activities [20]. However, the pharmacological effects of the combination of the ESM, AJN, and AJK extracts have not been demonstrated previously. Thus, in the present study, the beneficial effects of the nuruk fermented extract mix, Vigeo, on bone metabolism were studied for the first time.

In this study, we investigated the effect of Vigeo on the lipopolysaccharide (LPS)triggered bone loss model by measuring bone loss, osteoclast activation, and bone resorption content. To support the promising in vivo effect of Vigeo, we also examined the inhibitory effects of Vigeo on receptor activator of nuclear factor-B ligand (RANKL)stimulated osteoclast activation and the underlying mechanisms in vitro.

\section{Materials and Methods}

\subsection{Preparation of Vigeo}

Vigeo ${ }^{\circledR}$ was supplied by PANAX BIO (Panax Bio Co., Ltd., Nonsan, Korea). Briefly, Vigeo was manufactured as follows: well washed and dried Eleutherococcus senticosus Maxim (135 g), Achyranthes japonica (Miq.) Nakai (78 g), and Atractylodes japonica Koidzumi (78 g) were extracted with hot water for 180 min using a Kyungseo extractor (COSMOS-660, Kyungseo E\&P Co., Ltd., Incheon, Korea). Fresh yeast, rich nuruk, and popped rice were prepared for nuruk fermentation. After mixing rice nuruk $(1 \mathrm{~kg})$ with fresh yeast $(4 \mathrm{~g})$ and distilled water $(1.5 \mathrm{~L})$, the mixture was fermented for $96 \mathrm{~h}$. Then, popped rice $(3 \mathrm{~kg})$ and a combined extract (hot water extract) were mixed and fermented at $26^{\circ} \mathrm{C}$ for 15 days. The nuruk fermented extract mixture (Vigeo) was freeze-dried and then dissolved in distilled water according to the required concentration.

\subsection{Reagents and Antibodies}

Recombinant human RANKL and human macrophage colony-stimulating factor (MCSF) were purchased from PeproTech EC, Ltd. (London, UK). The $\beta$-actin antibody was obtained from Sigma-Aldrich (St. Louis, MO, USA). Specific antibodies against phosphop38, p38, phospho-ERK 1/2, ERK 1/2, phospho-JNK, JNK, phospho-IkB, phospho-AKT, and AKT, c-FOS, nuclear factor of activated T cells c1 (NFATC1), and IKB were purchased from Santa Cruz Biotechnology (Santa Cruz, CA, USA). Alpha minimal essential medium $(\alpha-M E M)$, fetal bovine serum (FBS), and penicillin-streptomycin solution were purchased from Gibco BRL (Grand Island, NY, USA).

\subsection{Mice Care and Ethics Statement}

The mice were tested by purchasing five-week-old males of the imprinting control region (ICR) mice (weighing $35 \pm 2 \mathrm{~g}$ ) from Samtako Co., Ltd. (Osan, Korea). All mice were fed a normal diet and provided free access to water, maintained at an ambient temperature of $22-24{ }^{\circ} \mathrm{C}$ and relative humidity of $55-60 \%$ under a $12 \mathrm{~h}: 12 \mathrm{~h}$ light/dark cycle in a pathogen-free environment. Mouse studies were approved by the Institutional Animal 
Care and Use Committee (IACUC) of Wonkwang University (Permit number: WKU-21-02), Republic of Korea, and conducted as per the standard guidelines. The mice were monitored daily to check their health status.

\subsection{LPS-Mediated Bone Loss Mouse Model}

Five-week-old male ICR mice were randomly divided into four groups: control $(n=6)$, LPS $(5 \mathrm{mg} / \mathrm{kg})$ treated $(n=6)$, LPS $(5 \mathrm{mg} / \mathrm{kg})+$ Vigeo $(100 \mathrm{mg} / \mathrm{kg})$ treated $(n=6)$, and LPS $(5 \mathrm{mg} / \mathrm{kg})+$ Vigeo $(200 \mathrm{mg} / \mathrm{kg})$ treated $(n=6)$. Vigeo or PBS was administered orally every 9 days before the first LPS injection. Mice were intraperitoneally injected with LPS $(10 \mathrm{mg} / \mathrm{kg})$ or an equal volume of PBS on days 2 and 6 . The mice were weighed daily. On the 9th day, all mice were euthanized, and their femurs and bloods were harvested for subsequent analysis.

\subsection{Micro-Computed Tomography (Micro-CT) and Histopathology}

The isolated femurs were fixed with $4 \%$ paraformaldehyde for one day. The distal metaphysis of the femur was analyzed by high-resolution micro-CT (NFR-Polaris-S160; Nanofocus Ray, Iksan, Korea) with a source voltage of $45 \mathrm{kVp}, 90 \mu \mathrm{A}$ current, and $7 \mu \mathrm{m}$ isotropic resolution. Morphometric parameters, including bone volume per tissue volume $(\mathrm{BV} / \mathrm{TV})$, trabecular separation (Tb. Sp), trabecular thickness (Tb. Th), and trabecular number $(\mathrm{Tb} . \mathrm{N})$ were individually calculated using INFINITT-Xelis software 1.16 (INFINITT Healthcare, Seoul, Korea). For the bone histological analysis, femurs were dissected and fixed in $4 \%$ paraformaldehyde in PBS for $48 \mathrm{~h}$. Femurs were then decalcified by placing them for two weeks in $12 \%$ EDTA, which was replaced every three days. The tissues were dehydrated, embedded in paraffin, and cut to a thickness of $6 \mu \mathrm{m}$ along the coronal plate. The decalcified femoral sections were stained with hematoxylin and eosin (H\&E) and tartrate-resistant acid phosphatase (TRAP).

\subsection{Enzyme-Linked Immunosorbent Assay (ELISA)}

Serum was collected from mice by cardiac puncture followed by centrifugation at $12,000 \times g$ for $15 \mathrm{~min}$. The serum level of the bone resorption marker C-terminal telopeptide of type I collagen (CTX-I) was measured using ELISA kits (Mybiosource Inc., San Diego, CA, USA), according to the manufacturer's instructions.

\subsection{Preparation of Mouse Bone Marrow Macrophages (BMMs) and Osteoclast Differentiation}

Bone marrow cells (BMCs) were isolated from 5-week-old ICR mice by flushing the tibias and femurs with growth medium $\alpha$-MEM. To obtain BMMs, BMCs were seeded on culture dishes in $\alpha$-MEM supplemented with $10 \%$ FBS and $10 \mathrm{ng} / \mathrm{mL}$ M-CSF and cultured for one day. Non-adherent cells were transferred to $10-\mathrm{cm}$ petri dishes and further cultured in the presence of M-CSF $(30 \mathrm{ng} / \mathrm{mL})$ for three days. Cells attached to the bottom of the petri dishes were considered as BMMs. To differentiate BMMs into osteoclasts, BMMs were cultured in 48-well plates $\left(3.5 \times 10^{4}\right.$ cells/well) in the presence of M-CSF $(30 \mathrm{ng} / \mathrm{mL})$ and RANKL $(100 \mathrm{ng} / \mathrm{mL})$ and the activity was tested by treatment with Vigeo $(0,25$, $50,100 \mu \mathrm{g} / \mathrm{mL}$ ). After 3-4 days, the differentiated cells were fixed with $3.7 \%$ formalin for $15 \mathrm{~min}$, permeabilized with $0.1 \%$ Triton X-100 for $10 \mathrm{~min}$, and stained with a TRAP staining solution. The stained cells were observed under a microscope, and the number of TRAP-positive multinucleated cells (TRAP + MNCs) was counted.

\subsection{Evaluation of Cytotoxicity}

To determine cell viability by XTT assay, BMMs were seeded in 96-well plates $\left(1 \times 10^{4}\right.$ cells/well) in the presence of M-CSF $(30 \mathrm{ng} / \mathrm{mL})$ with the indicated concentrations of Vigeo. Following 3-day incubation, XTT (sodium 30-[1-(phenyl-aminocarbonyl)-3, 4-tetrazolum]-bis(4-methoxy-6-nitro) benzenesulfonic acid hydrate and N-methyl dibenzopyrazine methyl sulfate) solution $(50 \mu \mathrm{L})$ was added into each well and incubated for $4 \mathrm{~h}$. 
Absorbance at $450 \mathrm{~nm}$ was measured using a multi-detection microplate reader (Molecular Devices, Sunnyvale, CA, USA).

\subsection{Quantitative Real-Time Polymerase Chain Reaction ( $q R T-P C R$ )}

Total RNA was extracted using TRIzol reagent (Thermo Fisher Scientific, Waltham, MA, USA) according to the manufacturer's instructions and quantified by measuring the absorbance at $260 \mathrm{~nm}$. Single-stranded cDNA was prepared from $1 \mu \mathrm{g}$ of total RNA using M-MLV reverse transcriptase with oligo-dT primers according to the manufacturer's instructions (Invitrogen, Carlsbad, CA, USA). Quantitative RT-PCR was carried out on the Exicycler 96 Real-Time Quantitative Thermal Block (Bioneer Co., Daejeon, Korea) using the SYBR ${ }^{\circledR \circledR}$ Green Premix (Bioneer Co., Daejeon, Korea) with specific primers. The amplification conditions were $95^{\circ} \mathrm{C}$ for $5 \mathrm{~min}$, followed by 40 cycles of $95^{\circ} \mathrm{C}$ for $1 \mathrm{~min}, 60^{\circ} \mathrm{C}$ for $30 \mathrm{~s}$, and $72{ }^{\circ} \mathrm{C}$ for $1 \mathrm{~min}$. Using Gapdh as an internal control, relative gene expression among samples was determined using the threshold cycle $\left(\mathrm{C}_{t}\right)$ value. The primer sequences are listed in Table 1.

Table 1. Primer sequences used for real-time qRT-PCR analysis.

\begin{tabular}{ccc}
\hline \multirow{2}{*}{ Gene Name } & \multicolumn{2}{c}{ Sequence $\mathbf{( 5}^{\prime} \mathbf{- 3}^{\prime}$ ) } \\
\cline { 2 - 3 } & Forward & Reverse \\
\hline Gapdh & TCAAGAAGGTGGTGAAGCAG & AGTGGGAGTTGCTGTTGAAGT \\
\hline c-Fos & GGTGAAGACCGTGTCAGGAG & TATTCCGTTCCCTTCGGATT \\
\hline Nfatc1 & GAGTACACCTTCCAGCACCTT & TATGATGTCGGGGAA AGAGA \\
\hline DC-stamp & TCCTCCATGAACAAACAGTTCCA & AGACGTGGTTTAGGAATGCAGCTC \\
\hline OC-stamp & ATGAGGACCATCAGGGCAGCCACG & GGAGAAGCTGGGTCAGTAGTTCGT \\
\hline$\alpha v$-integrin & ACAAGCTCACTCCCATCACC & ATATGAGCCTGCCGACTGAC \\
\hline$\beta_{3}$-integrin & GGAGTGGCTGATCCAGATGT & TCTGACCATCTTCCCTGTCC \\
\hline Atp6v0d2 & GACCCTGTGGCACTTTTTGT & GTGTTTGAGCTTGGGGAGAA \\
\hline Cathepsin $K($ Ctsk $)$ & CCAGTGGGAGCTATGGAAGA & CTCCAGGTTATGGGCAGAGA \\
\hline
\end{tabular}

\subsection{Filamentous-Actin (F-Actin) Assay}

To experiment with F-actin ring formation, BMMs were incubated with M-CSF (30 ng/mL) and RANKL (100 ng/mL) at different concentrations of Vigeo $(25,50$, and $100 \mu \mathrm{g} / \mathrm{mL}$ ). After the 3-day incubation, the cells were fixed in $3.7 \%$ formalin for $15 \mathrm{~min}$, permeabilized with $0.1 \%$ Triton $\mathrm{X}-100$ for $10 \mathrm{~min}$, with $0.25 \%$ bovine serum albumin (Sigma-Aldrich, St. Louis, MO, USA) for $30 \mathrm{~min}$, and stained with phalloidin and a 40,6diamidino-2-phenylindole (DAPI) solution (Life Technologies, Grand Island, NY, USA) to visualize F-actin and nuclei, respectively. Fluorescence was detected using a fluorescence microscope (Olympus FV1000, Tokyo, Japan) and images were analyzed using Image-Pro Plus software (version 4.0; Media Cybernetics, Silver Spring, MD, USA).

\subsection{Resorption Pit Assay}

The bone resorption assay was performed as described previously [21]. Briefly, mature osteoclasts were generated from the co-culture of BMCs and primary osteoblasts (OBs) in $\alpha$-MEM complete medium containing $10^{-8} \mathrm{M}$ Vitamin D3 and $10^{-6} \mathrm{M}$ prostaglandin E2 (Sigma-Aldrich) for 10-12 days on collagen gel-coated culture dishes. Mature OCs were obtained by treatment with $0.1 \%$ collagenase type IV (Sigma-Aldrich, St. Louis, MO) and were reseeded in 48-well plates and dentin slices in the presence or absence of Vigeo $(100 \mu \mathrm{g} / \mathrm{mL})$. The cells re-seeded in dentin slices were completely removed using $10 \%$ sodium hypochlorite after $48 \mathrm{~h}$. Subsequently, dentin slices were stained with hematoxylin. Bone slice images were taken using a microscope, and resorbed areas on bone slices were quantified with ImageJ software. 


\subsection{Western Blotting}

Cells incubated with the indicated reagents for the indicated durations were washed with cold PBS and lysed with cold lysis buffer containing $50 \mathrm{mM}$ Tris- $\mathrm{HCl}, 150 \mathrm{mM}$ $\mathrm{NaCl}, 5 \mathrm{mM}$ EDTA, $1 \%$ Triton $\mathrm{X}-100,1 \mathrm{mM}$ sodium fluoride, $1 \mathrm{mM}$ sodium vanadate, $1 \%$ deoxycholate, and protease inhibitors on ice for $30 \mathrm{~min}$. The lysate was collected after centrifugation at $10,000 \times g$ for $15 \mathrm{~min}$. The protein concentration of each sample was quantified using a Bio-Rad DC Protein Assay Kit (Bio-Rad Laboratories Inc., Hercules, CA, USA). Equal amounts of proteins (10-20 $\mu \mathrm{g}$ ) were separated by $10 \%$ SDS-PAGE, transferred to PVDF membranes (Bio-Rad, Hercules, CA), blocked with 5\% nonfat milk in TBST buffer (100 mM NaCl, $10 \mathrm{mM}$ Tris-HCl, $\mathrm{pH} \mathrm{7.5,} \mathrm{and} \mathrm{0.1 \%} \mathrm{Tween-20)} \mathrm{for} 1 \mathrm{~h}$ at room temperature, and incubated with specific primary antibodies overnight at $4{ }^{\circ} \mathrm{C}$. Finally, the membranes were incubated for $1 \mathrm{~h}$ at room temperature with the secondary antibodies. Following extensive washing in TBST, the specific protein bands were visualized using Immobilon Western Chemiluminescent HRP Substrate (Millipore, Billerica, MA, USA). Actin was used as a loading control.

\subsection{Statistical Analysis}

All quantitative data are presented as the mean \pm standard deviation (SD) of at least three independent experiments. Statistical analysis was performed using Student's $t$-test for comparisons between two groups and analysis of variance (ANOVA) followed by Tukey's posthoc test for comparisons among three groups using GraphPad Prism 5 (GraphPad Software, San Diego, CA, USA). $p<0.05$ was considered as statistically significant.

\section{Results}

\subsection{Oral Administration of Vigeo Ameliorates LPS-Induced Bone Loss In Vivo}

First, we investigated the effects of Vigeo on an LPS-induced bone loss mouse model. Mice were intraperitoneally injected with LPS and orally administered Vigeo or PBS. As shown in Figure 1A, all the experimental animals showed a stable increase in body weight during 9 days, demonstrating no biotoxicity at the Vigeo-treated concentration. As shown in Figure 1B, micro-CT analysis was performed using dissected femurs, which showed that LPS-induced mice developed a significant osteoporosis phenotype in femoral trabecular bone compared with those in the control group. In contrast, the group that was orally administered $200 \mathrm{mg} / \mathrm{kg}$ of Vigeo significantly reduced femoral trabecular bone loss in the LPS plus Vigeo group compared with that in the LPS alone group, which preserved the partial recovery of bone. Quantification of bone parameters verified that $200 \mathrm{mg} / \mathrm{kg}$ Vigeo administration significantly increased bone mineral density, such as BV/TV, Tb. N, and Tb. Th; whereas, Tb. Sp was decreased in LPS-induced bone loss mouse models (Figure 1C). In addition, the LPS plus Vigeo $100 \mathrm{mg} / \mathrm{kg}$ group also showed an inhibitory effect on bone loss but was not statistically significant. Taken together, the in vivo results indicated that Vigeo demonstrated considerable efficacy in rescuing bone loss.

\subsection{Vigeo Effectively Attenuates LPS-Induced Osteoclast Activation In Vivo}

Next, we confirmed the protective effect of Vigeo on histological changes of LPSinduced bone through H\&E and TRAP staining. H\&E staining showed severe bone loss and reduced bone volume with abnormal trabecular structures in the LPS-treated group (Figure 2A). However, treatment with Vigeo restored trabecular bone volume in the $200 \mathrm{mg} / \mathrm{kg}$ Vigeo-treated groups, showing restored trabecular density and preservation of bone structure (Figure 2A). Furthermore, TRAP staining results demonstrated that the LPS-treated group remarkably increased the number of TRAP-positive osteoclasts, while Vigeo attenuated LPS-induced osteolytic lesions, with decreased numbers of TRAP-stained mature osteoclasts (Figure 2B,C). As shown in Figure 2D, significant increase of CTX-I levels was observed in the LPS-induced group compared with the control group. Nonetheless, treatment with Vigeo reduced this tendency, as evidenced by the gradual decrease in CTX-I 
levels following Vigeo administration. These results reflect the effective anti-osteoclast activation effects of Vigeo in vivo.

A

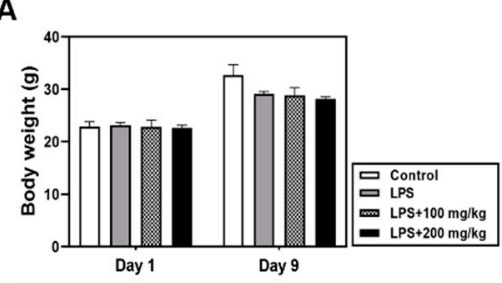

B

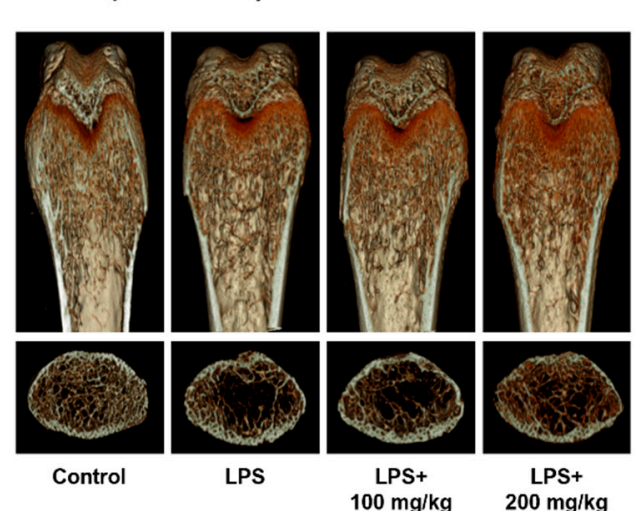

C
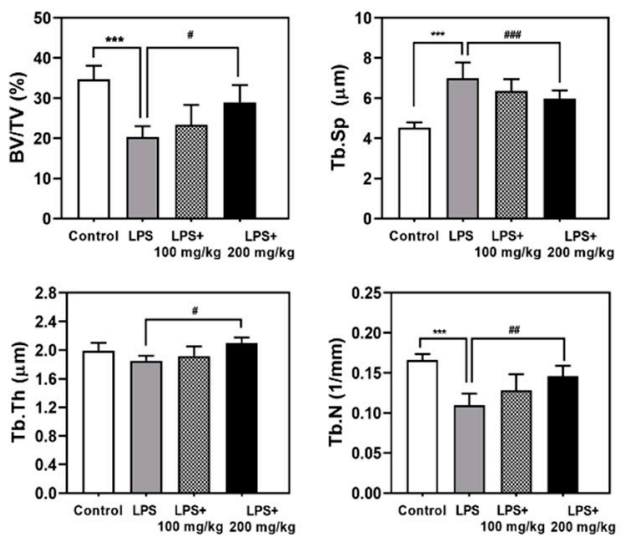

Figure 1. Vigeo attenuates lipopolysaccharide (LPS)-induced inflammatory bone loss. Mice were intraperitoneally injected with LPS $(10 \mathrm{mg} / \mathrm{kg})$ followed by treatment with indicated concentrations of Vigeo for 7 days. (A) Body weight measurement during the experimental period. (B) Representative three-dimensional micro-computed tomography (micro-CT) images of the coronal and transverse sections in the distal femurs of a mouse from each group. (C) Measurement of the trabecular morphometric parameters of bone volume/total volume (BV/TV), trabecular separation ( $\mathrm{Tb}$. Sp), trabecular thickness (Tb. $\mathrm{Th}$ ), and trabecular number ( $\mathrm{Tb} . \mathrm{N}$ ) in the proximal femurs using the micro-CT data using the INFINITT-Xelis software 1.16. Statistical analysis was performed using one-way analysis of variance (ANOVA) followed by Tukey's posthoc test for comparisons among three groups. ${ }^{* * *} p<0.01$ versus the control group; ${ }^{\#} p<0.05,{ }^{\# \#} p<0.01$, ${ }^{\# \#} p<0.001$ versus the LPS group.

\subsection{Vigeo Inhibits Osteoclast Differentiation and Bone Resorption In Vitro}

In order to prevent Vigeo-induced toxicity in primary BMM cells, we determined the nontoxic concentrations of Vigeo against preosteoclasts to observe its anti-osteoclastogenesis effects. The XTT assay results showed that Vigeo had no significant effect on cell viability at the indicated concentrations (Figure 3A). Next, we assessed the anti-osteoclastogenetic effect of Vigeo by culturing BMM cells with RANKL and M-CSF in the presence and absence of Vigeo and then examined them after TRAP staining. The results showed the formation of osteoclasts in control groups after RANKL stimulation, whereas the addition of Vigeo remarkably delayed the differentiation of osteoclasts in a dose-dependent manner (Figure 3B,C), indicating that nontoxic levels of Vigeo were able to significantly suppress osteoclast formation. Osteoclast function requires a well-formed F-actin rings, which is consistent with the results shown in Figure 4A,B. Upon stimulation with RANKL, BMMs showed the generation of the characteristically polarized F-actin ring. However, treatment with Vigeo markedly diminished the number and size of F-actin rings, demonstrating inhibited osteoclast activation (Figure 4A,B). To further test the effects of Vigeo on RANKL-induced osteoclast bone resorbing function, we performed a pit formation assay on dentin slices. As shown in Figure 4C, Vigeo treatment effectively decreased the number of resorption pits. These results collectively demonstrate that Vigeo reduced the formation and bone resorption properties of osteoclasts without cytotoxic effects. 
A
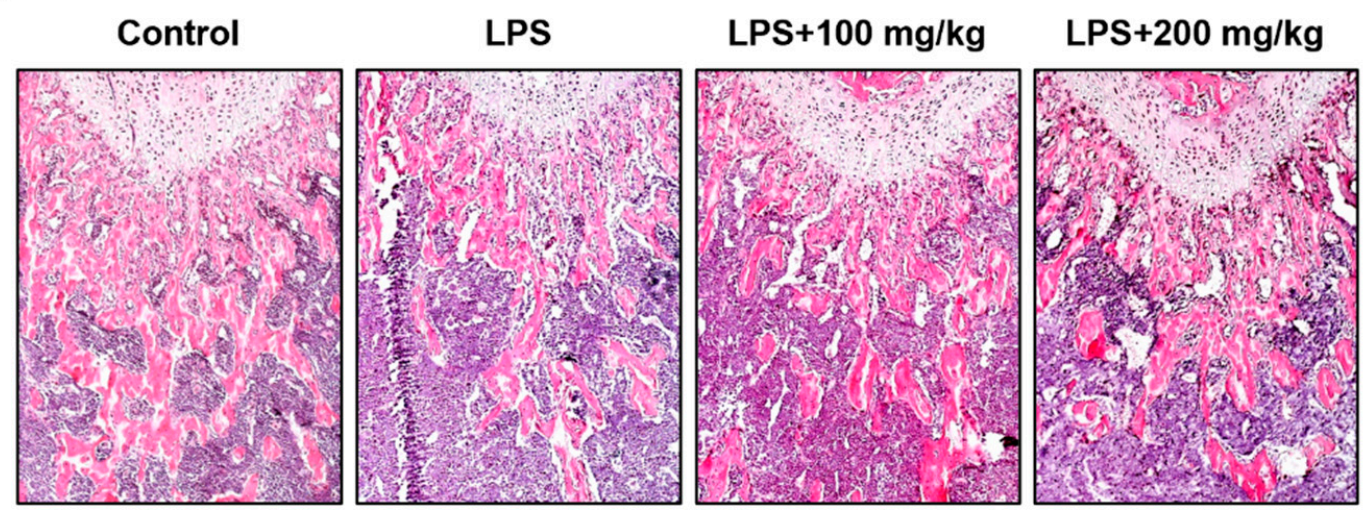

B

Control

\section{LPS}
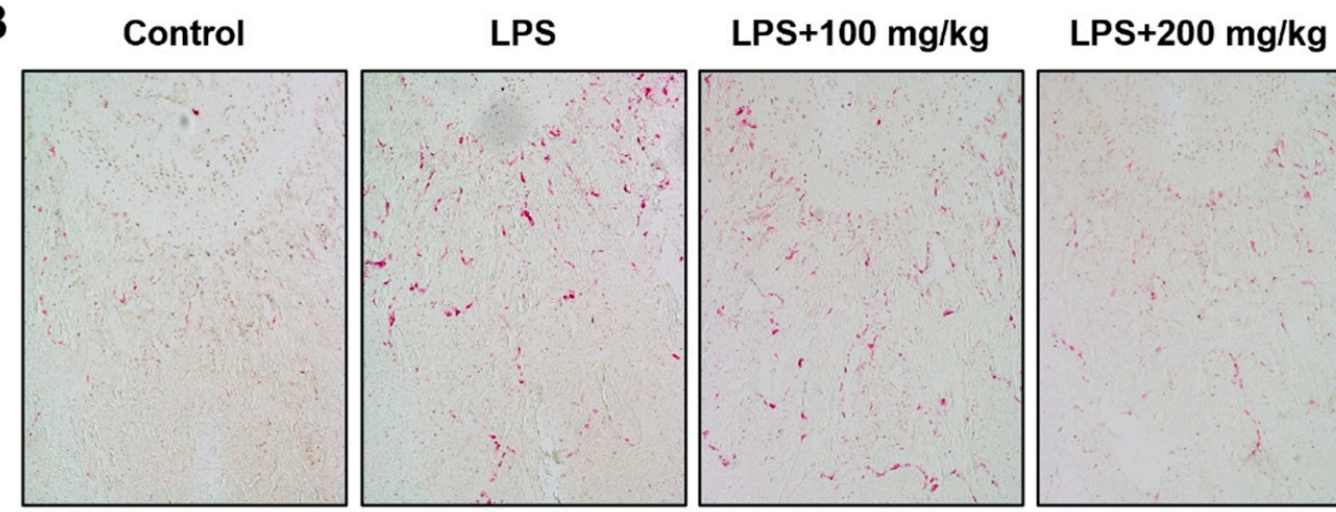

C

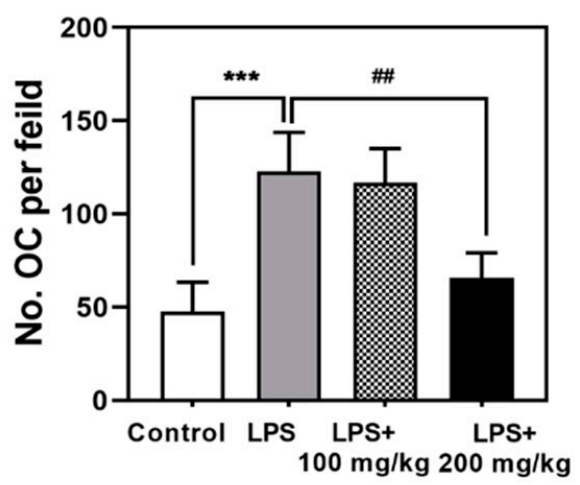

D

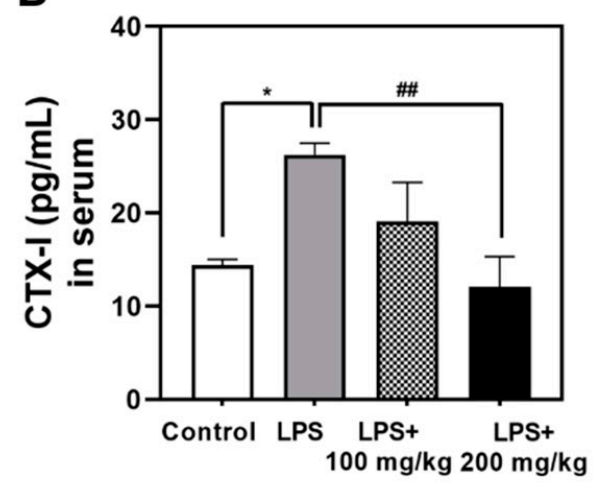

Figure 2. Vigeo inhibits lipopolysaccharide (LPS)-induced osteoclast activation in mice. (A) LPS injected mice were sacrificed after 9 days, and dissected femurs were fixed, decalcified, paraffin-embedded, and sectioned. The slices were stained with hematoxylin and eosin (H\&E). (B) Slices stained with tartrate-resistant acid phosphatase (TRAP). (C) The number of osteoclasts per visual field of tissue was measured by histomorphometric analysis. (D) Blood samples were collected from mice and centrifuged to obtain serum for the evaluation of CTX-I levels by ELISA. Statistical analysis was performed using one-way analysis of variance (ANOVA) followed by Tukey's posthoc test for comparisons among the three groups. ${ }^{*} p<0.05,{ }^{* * *} p<0.001$ versus the control group; ${ }^{\# \#} p<0.01$ versus the LPS group. 
A

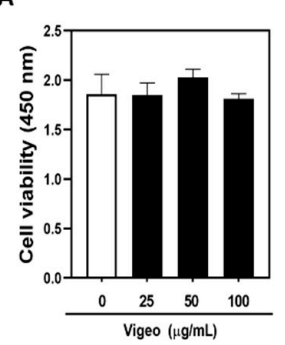

B

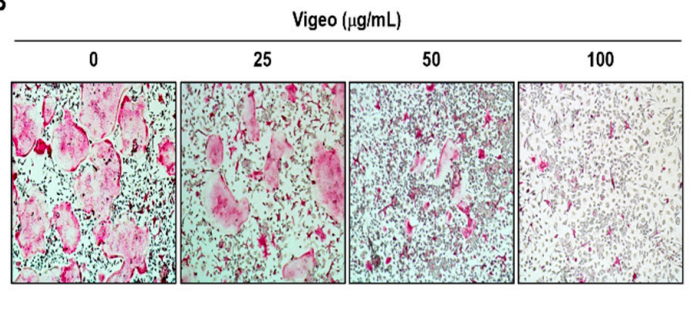

C

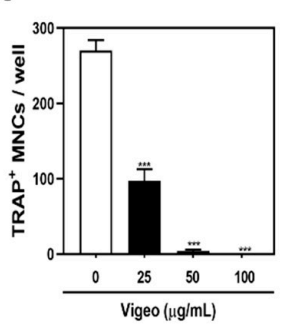

Figure 3. Vigeo attenuates receptor activator of nuclear factor-B ligand (RANKL)-induced osteoclast differentiation without cytotoxicity. (A) Bone marrow macrophages (BMMs) were cultured with $30 \mathrm{ng} / \mathrm{mL} \mathrm{M}-\mathrm{CSF}$ and the indicated concentration of Vigeo for 3 days. Cell viability of Sec was evaluated using the XTT assay by measuring absorbance at $450 \mathrm{~nm}$. (B) BMMs were seeded with M-CSF (30 ng/mL) and stimulated with RANKL (100 ng/mL) and the indicated concentrations of Vigeo for 3 days. TRAP staining was performed to assess osteoclast formation and representative images were captured. (C) TRAP-positive multinucleated cells counted as osteoclasts (nuclei $>5$ ). ${ }^{* * *} p<0.001$ versus the control group.
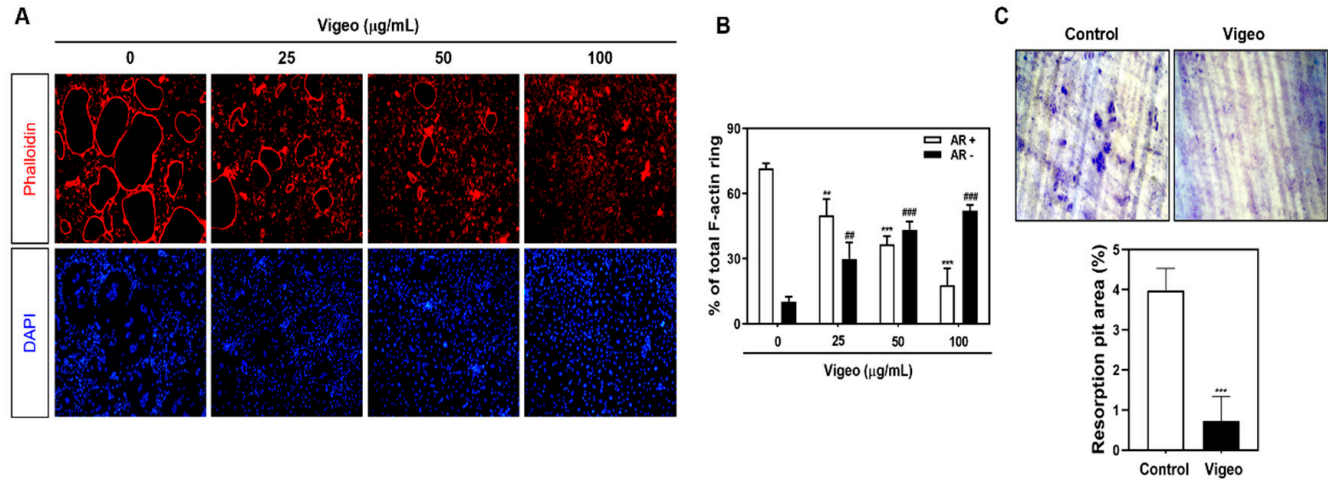

Figure 4. Vigeo suppresses the F-actin ring formation and bone-resorbing activity of mature osteoclasts. (A) Bone marrow macrophages (BMMs)were cultured with M-CSF $(30 \mathrm{ng} / \mathrm{mL})$ and receptor activator of nuclear factor-B ligand (RANKL) $(100 \mathrm{ng} / \mathrm{mL})$ in the presence of the indicated concentrations of Vigeo. The cells were fixed, permeabilized, and stained with phalloidin and 4',6-diamidino-2-phenylindole (DAPI). The cells were examined under a confocal laser scanning microscope. (B) Normal actin ring $(\mathrm{AR}+)$ osteoclasts and disrupted actin ring $(\mathrm{AR}-)$ osteoclasts were counted and compared with the total number of osteoclasts that contain three or more nuclei. The graph shows the comparison between relative percentage of osteoclasts that express $\mathrm{AR}+$ and $\mathrm{AR}-$. $^{* *} p<0.01$ and ${ }^{* * *} p<0.001$ versus $\mathrm{AR}+$ osteoclasts in control group; ${ }^{\# \#} p<0.01$ and ${ }^{\# \#} p<0.001$ versus AR - osteoclasts in control group. (C) Mature osteoclasts from the co-culture system were seeded in dentin slices for $48 \mathrm{~h}$ with or without Vigeo $(100 \mu \mathrm{g} / \mathrm{mL})$. Cells attached to the hydroxyapatite-coated plate were removed, and the plates were photographed under a light microscope. ${ }^{* * *} p<0.001$ versus the control.

\subsection{Vigeo Inhibits RANKL-Activated Osteoclast Differentiation Signaling Pathways and Osteoclast Specific Genes In Vitro}

To elucidate the upstream mechanisms by which Vigeo affects osteoclast activation, we investigated the AKT, IKB, and MAPK (JNK, p38, and ERK) signaling pathways, and their downstream molecules NFATc-1 and c-FOS. As shown in Figure 5A, increased phosphorylation of AKT, JNK, p38, ERK, and IKB mediated by RANKL stimulation was significantly blocked by Vigeo. Next, to evaluate whether Vigeo affect the induction of $c$-Fos, Nfatc1, and osteoclast-specific genes, we examined their mRNA and protein levels. As shown in Figure 5B, Vigeo suppressed RANKL-induced expression of $c$-Fos and NfatC1 mRNA. In agreement with these results, the protein levels of $c$-Fos and Nfatc1 induced by RANKL were significantly increased, and this increase was significantly inhibited by Vigeo (Figure 5C). In addition, Vigeo also inhibited the RANKL-induced increase in the mRNA expression of DC-cstamp, OC-stamp, $\alpha v$-integrin, $\beta_{3}$-integrin, Atp6v0d2, and Ctsk at $48 \mathrm{~h}$ (Figure 6). These results demonstrate that Vigeo inhibited RANKL-induced osteoclastogenesis through de-phosphorylation of AKT, JNK, p38, ERK, and IKB, followed by downregulation of transcription factors. 

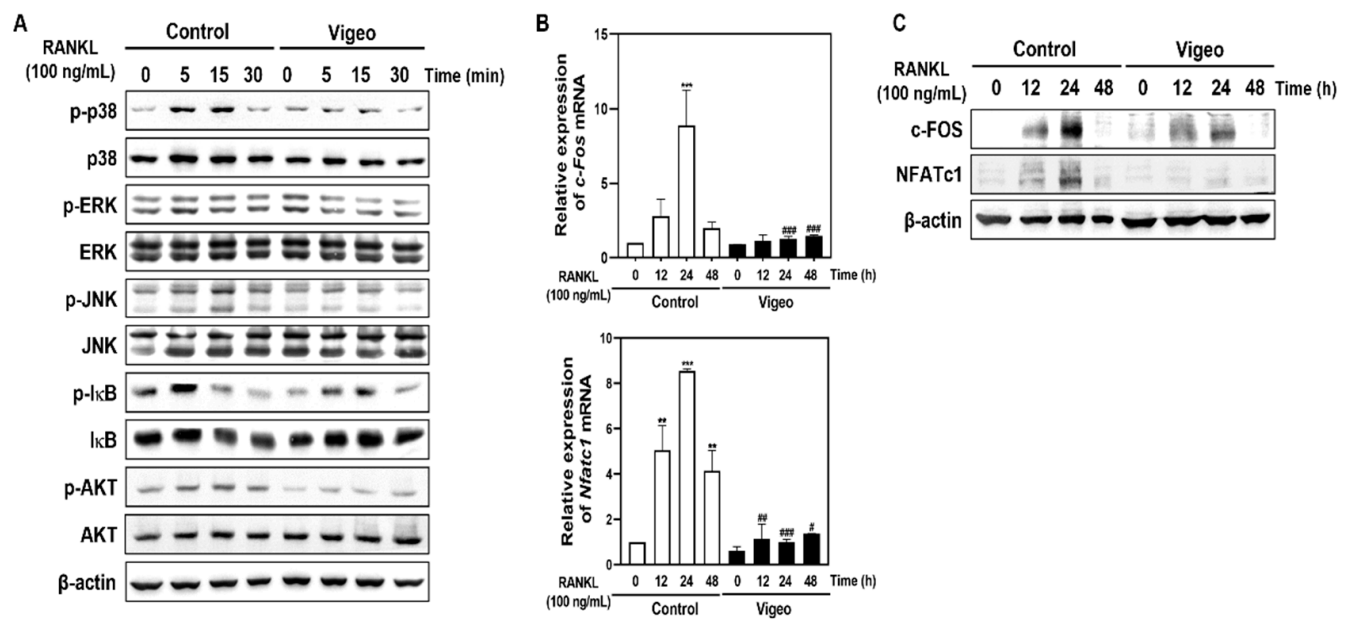

Figure 5. Vigeo affects receptor activator of nuclear factor-B ligand (RANKL)-induced phosphorylation of early signals and expression of c-FOS and NFATc1. (A) Bone marrow macrophages (BMMs)were seeded in the presence of M-CSF $(10 \mathrm{ng} / \mathrm{mL}$ ) for 1 day and then starved for $4 \mathrm{~h}$. (A) Vigeo was used to pretreat the cells for $1 \mathrm{~h}$ followed by $0,5,15$, and 30 min of RANKL stimulation. Whole-cell proteins were extracted by lysis using RIPA buffer and the protein expression was detected by western blotting using the indicated antibodies. (B) BMMs were cultured with M-CSF ( $30 \mathrm{ng} / \mathrm{mL})$ and RANKL $(100 \mathrm{ng} / \mathrm{mL})$ in the presence or absence of Vigeo $(100 \mu \mathrm{g} / \mathrm{mL})$. Total RNA was isolated from the cells and mRNA expression levels of $c$-Fos and Nfatc1 were evaluated by real-time RT-PCR. ${ }^{* *} p<0.01,{ }^{* * *} p<0.001$ versus the control; ${ }^{\#} p<0.05,{ }^{\# \#} p<0.01,{ }^{\# \#} p<0.001$ versus the control at indicated times. (C) BMMs were cultured with M-CSF (30 ng/mL) and RANKL $(100 \mathrm{ng} / \mathrm{mL})$ in the presence or absence of Vigeo $(100 \mu \mathrm{g} / \mathrm{mL})$. Protein expression was detected using western blotting with the indicated antibodies. $\beta$-actin was used as an internal control.
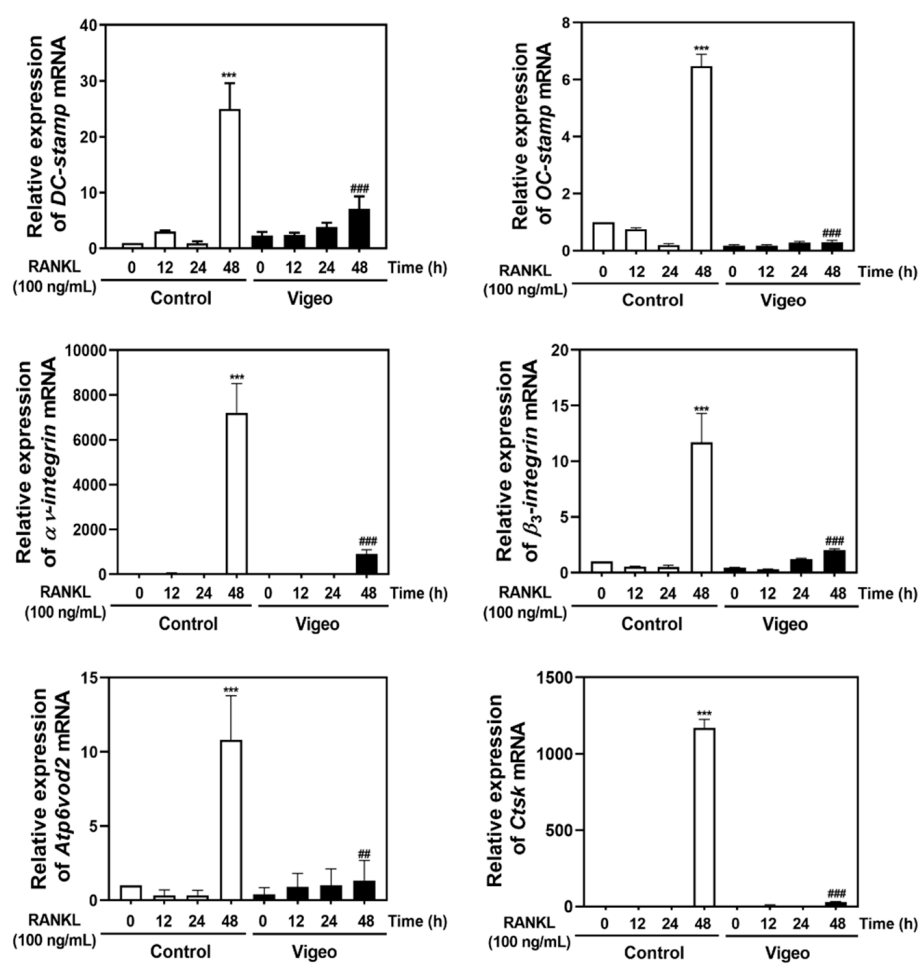

Figure 6. Vigeo inhibits receptor activator of nuclear factor-B ligand (RANKL)-induced mRNA expression of DC-stamp, OC-stamp, $\alpha v$-integrin, $\beta_{3}$-integrin, Atp6v0d2, and CtsK. Bone marrow macrophages (BMMs)were incubated with M-CSF (30 ng/mL) and RANKL $(100 \mathrm{ng} / \mathrm{mL})$ in the presence or absence of Vigeo $(100 \mu \mathrm{g} / \mathrm{mL})$ for the indicated time. Total RNA was isolated from cells using TRIzol reagent, and the mRNA expression of DC-stamp, OC-stamp, $\alpha v$-integrin, $\beta_{3}$-integrin, Atp6v0d2, and CtsK was analyzed by real-time RT-PCR. ${ }^{* * *} p<0.001$ versus the control at $0 \mathrm{~h}$; ${ }^{\# \#} p<0.01,{ }^{\# \#} p<0.001$ versus the control at $48 \mathrm{~h}$. 


\section{Discussion}

Fermentation is a biological system that depended on the microbial conversion of complex structures into simple compound by microorganisms such as bacteria, yeast, and fungi [8]. Fermentation-mediated bioactivation of plant medicinal products results in improved therapeutic efficacy and decreased toxicity [8,22], and fermentation is also known to have beneficial effects on the absorption and bioavailability of plant products by facilitating the production of active components into their metabolites or by generating lowmolecular-weight substances such as aglycones from glycosides $[23,24]$. These substances have diverse biological activities, such as anti-inflammatory activity, anti-infection, and anti-cancer properties $[25,26]$. Nuruk is a traditional Korean fermentation starter used to produce alcoholic beverages using various grains [27]. Nuruk contains different types of microorganisms, which are responsible for saccharification and alcoholic fermentation [27]. Recent studies have demonstrated that treatment with Nuruk extract is associated with a decrease in LPS-induced nitrite and IL-6 levels in RAW 264.7 cells [28], and also has an inhibitory effect on hypertension, migration, platelet aggregation, and angiogenesis [29].

In this study, we demonstrated for the first time that Vigeo, a functional extract using the traditional nuruk fermentation method, can inhibit RANKL-induced osteoclastogenesis by blocking the AKT, NF- $\mathrm{KB}$ and MAPK signaling pathways in vitro and prevent bone loss in vivo in an LPS-triggered bone loss mouse model. LPS-treated mice showed inflammatory bone loss by stimulating osteoclast differentiation, while oral-administration of Vigeo in LPS-treated mice prevented these effects. The micro-CT analysis results showed that LPStreated mice had suppressed BV/TV ratio, increased Tb. Sp $(\mu \mathrm{m})$, and suppressed $\mathrm{Tb} . \mathrm{N}$ (per $\mathrm{mm}$ ) and $\mathrm{Tb}$. Th $(\mu \mathrm{m})$; the effects that were significantly reversed by Vigeo treatment. In addition, the histological features were improved by Vigeo treatment in LPS-treated mice, and the LPS-treated TRAP-positive osteoclast count was also suppressed by Vigeo. Furthermore, Vigeo suppressed the levels of the bone resorption marker CTX-1 in the serum of LPS-treated mice.

Monocytes and macrophages derived from hematopoietic stem cells of the bone marrow are differentiated during the maturation step of osteoclastogenesis (differentiation, proliferation, polarization, and resorption) to generate osteoclasts. This process is regulated by the major cytokines M-CSF and RANKL, which differentiate BMM osteoclast precursors into osteoclasts [30]. Through TRAP staining and measuring the levels of bone resorption activity, such as the formation of F-actin and resorption pits, we determined that osteoclastogenesis and differentiation function can be effectively induced by co-stimulation with M-CSF and RANKL. The present study shows that non-toxic concentrations of Vigeo could inhibit large number of RANKL-stimulated TRAP-positive osteoclasts, demonstrating that Vigeo showed effective inhibitory effects against RANKL-induced osteoclastogenesis. Furthermore, the bone resorption activity, as measured by the materialization of F-actin ring structures, was also suppressed by Vigeo via inhibition of the formation of resorption pits formed by RANKL stimulation. These findings were further demonstrated by the reduced formation of F-actin rings and bone resorption pits which allow us to further explore the underlying mechanisms and to identify new molecular targets for the treatment of osteoporosis in the future.

Previous studies have revealed the biological effects of ESM extracts, a major component of Vigeo, including anti-inflammatory [10], anti-tumor [31], anti-steatosis [32], and neuroprotective [33] effects. Specifically, ESM extracts were found to inhibit MAPK and NF- $\mathrm{kB}$ signaling pathways, which are closely associated with osteoclast formation [12]. In the present study, Vigeo inhibited the phosphorylation of p38, ERK, JNK, IKB, and AKT in osteoclast precursor cells. Altogether, these results indicate that Vigeo could suppress the activities of $c$-Fos and Nfatc1 along with other transcriptional regulators of osteoclastogenesis. In addition, the regulation of $c$-Fos and Nfatc1 by Vigeo during osteoclastogenesis is promoted by the activity of osteoclast-specific genes such as DC-stamp, OC-stamp, $\alpha$ vintegrin, $\beta_{3}$-integrin, Atp6v0d2, and Ctsk. In future research, we will carry out a comparative analysis of the activity of a mixture of ESM, AJN, and AJK, which are constituents of 
Vigeo, and traditional nuruk fermented extract. Moreover, we will conduct isolation, purification, and analysis of components of Vigeo, which consists of various physiologically active substances.

\section{Conclusions}

Our findings show that Vigeo protects against LPS-induced inflammatory bone loss by inhibiting osteoclast activity in vivo. In vitro mechanistic studies demonstrated that Vigeo inhibits not only osteoclast differentiation but also F-actin formation and bone resorption through the inhibition of MAPK, NF- $\mathrm{kB}$, and AKT signaling, which leads to $c$-Fos and $N f a t c 1$ activation and expression of downstream genes. Although additional preclinical studies and clinical trials are needed to evaluate safety and efficacy in human, this study is the first to support the use of Vigeo as a promising treatment for the prevention of inflammatory bone diseases.

Author Contributions: Conceptualization, J.-Y.K. and M.S.L.; methodology, Y.-H.C. and S.Y.E.; software, C.H.C. and C.H.L.; validation, J.-Y.K., Y.-H.C., and S.Y.E.; formal analysis, Y.-H.C. and G.D.P.; investigation, J.-Y.K. and M.S.L.; resources, J.-Y.K.; data curation, S.Y.E., Y.-H.C., C.H.C. and C.H.L.; writing —original draft preparation, J.-Y.K., S.Y.E. and Y.-H.C.; writing—review and editing, J.-Y.K. and M.S.L.; visualization, G.D.P. and C.H.L.; supervision, M.S.L.; project administration, M.S.L.; funding acquisition, M.S.L. All authors have read and agreed to the published version of the manuscript.

Funding: This research was supported by a grant from the Korea Health Technology R\&D Project through the Korea Health Industry Development Institute (KHIDI), funded by the Ministry of Health \& Welfare, Republic of Korea (grant number: HI20C1951).

Institutional Review Board Statement: Mouse studies were approved by the Institutional Animal Care and Use Committee (IACUC) of Wonkwang University (Permit number: WKU-21-02), Republic of Korea, and conducted as per the standard guidelines.

Informed Consent Statement: Not applicable.

Data Availability Statement: The data used to support the finding of this study are available from the corresponding author upon request.

Conflicts of Interest: The authors declare no conflict of interest.

\section{References}

1. Kanis, J.A.; Melton, L.J.; Christiansen, C.; Johnston, C.C.; Khaltaev, N. The diagnosis of osteoporosis. J. Bone Miner. Res. 1994, 9, 1137-1141. [CrossRef] [PubMed]

2. Rousseau, S.; Kyomugasho, C.; Celus, M.; Hendrickx, M.E.G.; Grauwet, T. Barriers impairing mineral bioaccessibility and bioavailability in plant-based foods and the perspectives for food processing. Crit. Rev. Food Sci. Nutr. 2020, 60, 826-843. [CrossRef] [PubMed]

3. Xutian, S.; Zhang, J.; Louise, W. New exploration and understanding of traditional Chinese medicine. Am. J. Chin. Med. 2009, 37, 411-426. [CrossRef] [PubMed]

4. Cheon, Y.H.; Baek, J.M.; Park, S.H.; Ahn, S.J.; Lee, M.S.; Oh, J.M.; Kim, J.Y. Stauntonia hexaphylla (Lardizabalaceae) leaf methanol extract inhibits osteoclastogenesis and bone resorption activity via proteasome-mediated degradation of c-Fos protein and suppression of NFATc1 expression. BMC Complementary Altern. Med. 2015, 15, 280. [CrossRef]

5. Lim, D.W.; Kim, J.G.; Lee, Y.S.; Cha, S.H.; Kim, Y.T. Preventive Effects of Eleutherococcus senticosus Bark extract in OVX-induced osteoporosis in rats. Molecules 2013, 18, 7998-8008. [CrossRef]

6. Rizzello, C.G.; Coda, R.; Macías, D.S.; Pinto, D.; Marzani, B.; Filannino, P.; Giuliani, G.; Paradiso, V.M.; Di Cagno, R.; Gobbetti, M. Lactic acid fermentation as a tool to enhance the functional features of Echinacea spp. Microb. Cell Fact. 2013, 12, 40. [CrossRef] [PubMed]

7. Lei, V.; Amoa-Awua, W.K.A.; Brimer, L. Degradation of cyanogenic glycosides by Lactobacillus plantarum strains from spontaneous cassava fermentation and other microorganisms. Int. J. Food Microbiol. 1999, 53, 169-184. [CrossRef]

8. Parvez, S.; Malik, K.A.; Ah Kang, S.; Kim, H.Y. Probiotics and their fermented food products are beneficial for health. J. Appl. Microbiol. 2006, 100, 1171-1185. [CrossRef] [PubMed]

9. Hwang, Y.C.; Jeong, I.K.; Ahn, K.J.; Chung, H.Y. The effects of Acanthopanax senticosus extract on bone turnover and bone mineral density in Korean postmenopausal women. J. Bone Miner. Metab. 2009, 27, 584-590. [CrossRef] 
10. Jung, H.J.; Park, H.J.; Kim, R.G.; Shin, K.M.; Ha, J.; Choi, J.W.; Kim, H.J.; Lee, Y.S.; Lee, K.T. In vivo anti-inflammatory and antinociceptive effects of liriodendrin isolated from the stem bark of Acanthopanax senticosus. Planta Med. 2003, 69, 610-616.

11. Kropotov, A.V.; Kolodnyak, O.L.; Koldaev, V.M. Effects of Siberian ginseng extract and ipriflavone on the development of glucocorticoid-induced osteoporosis. Bull. Exp. Biol. Med. 2002, 133, 252-254. [CrossRef] [PubMed]

12. Yang, X.; Chang, Z.; Ma, R.; Guo, H.; Zhao, Q.; Wang, X.; Kong, L.; Hao, D. Eleutherococcus senticosus inhibits RANKL-induced osteoclast formation by attenuating the NF-KB and MAPKs signaling pathway. Int. J. Clin. Exp. Pathol. 2017, 10, 4514-4521.

13. Lee, S.G.; Lee, E.J.; Park, W.D.; Kim, J.B.; Kim, E.O.; Choi, S.W. Anti-inflammatory and anti-osteoarthritis effects of fermented Achyranthes japonica Nakai. J. Ethnopharmacol. 2012, 142, 634-641. [CrossRef] [PubMed]

14. Kim, D.; Park, K.K.; Lee, S.K.; Lee, S.E.; Hwang, J.K. Cornus kousa F. Buerger ex Miquel increases glucose uptake through activation of peroxisome proliferator-activated receptor $\gamma$ and insulin sensitization. J. Ethnopharmacol. 2011, 133, 803-809. [CrossRef] [PubMed]

15. Woo, Y.M.; Kim, O.J.; Jo, E.S.; Kim, S.J.; Lee, Y.H.; Ahn, M.Y.; Lee, S.H.; Ha, J.M.; Kim, A. Anti-inflammatory effects of the combined extracts of Achyranthes japonica nakai and Aralia continentalis kitagawa in vitro and in vivo. Data Brief 2019, 25, 104088. [CrossRef] [PubMed]

16. Zhao, X.; Kim, D.; Suminda, G.G.D.; Min, Y.; Yang, J.; Kim, M.; Zhao, Y.; Chosh, M.; Son, Y.O. Inhibitory effects of IL-6-mediated matrix metalloproteinase- 3 and -13 by Achyranthes japonica Nakai root in osteoarthritis and rheumatoid arthritis mice models. Pharmaceutical 2021, 14, 776.

17. Park, J.H.; Kang, S.N.; Shin, D.; Hur, I.C.; Kim, I.S.; Jin, S.K. Antioxidant activities of Achyranthes japonica Nakai extract and its application to the pork sausages. Asian-Australas. J. Anim. Sci. 2013, 26, 287-294. [CrossRef]

18. Hong, M.H.; Kim, J.H.; Bae, H.; Lee, N.Y.; Shin, Y.C.; Kim, S.H.; Ko, S.G. Atractylodes japonica koidzumi inhibits the production of proinflammatory cytokines through inhibition of the NF-kappaB/IkappaB signal pathway in HMC-1 human mast cells. Arch. Pharm. Res. 2010, 33, 843-851. [CrossRef]

19. Wang, K.T.; Chen, L.G.; Chou, D.S.; Liang, W.L.; Wang, C.C. Anti-oxidative abilities of essential oils from Atractylodes ovate rhizome. Evid. -Based Complementary Altern. Med. 2011, 2011, 204892. [CrossRef]

20. Han, Y.; Jung, H.W.; Park, Y.K. The roots of Atractylodes japonica Koidzumi promote adipogenic differentiation via activation of the insulin signaling pathway in 3T3-L1 cells. BMC Complementary Altern. Med. 2012, 12, 154. [CrossRef] [PubMed]

21. Kim, J.Y.; Cheon, Y.H.; Kwak, S.C.; Baek, J.M.; Yoon, K.H.; Lee, M.S.; Oh, J. Emodin regulates bone remodeling by inhibiting osteoclastogenesis and stimulating osteoblast formation. J. Bone Miner. Res. 2014, 29, 1541-1553. [CrossRef] [PubMed]

22. Lee, S.O.; Kim, S.J.; Kim, J.S.; Ji, H.; Lee, E.O.; Lee, H.J. Comparison of the main components and bioactivity of Rhus verniciflua Stokes extracts by different detoxification processing methods. BMC Complementary Altern. Med. 2018, 18, 242. [CrossRef]

23. Bae, E.A.; Hyun, Y.J.; Choo, M.K.; Oh, J.K.; Ryu, J.H.; Kim, D.H. Protective effect of fermented red ginseng on a transient focal ischemic rats. Arch. Pharm. Res. 2004, 27, 1136-1140. [CrossRef]

24. Joo, S.S.; Won, T.J.; Nam, S.Y.; Kim, Y.B.; Lee, Y.C.; Park, S.Y.; Park, H.Y.; Hwang, K.W.; Lee, D.I. Therapeutic advantages of medicinal herbs fermented with Lactobacillus plantarum, in topical application and its activities on atopic dermatitis. Phytother. Res. 2009, 23, 913-919. [CrossRef] [PubMed]

25. Ng, C.C.; Wang, C.Y.; Wang, Y.P.; Tzeng, W.S.; Shyu, Y.T. Lactic acid bacterial fermentation on the production of functional antioxidant herbal Anoectochilus formosanus Hayata. J. Biosci. Bioeng. 2011, 111, 289-293. [CrossRef] [PubMed]

26. Oh, Y.C.; Cho, W.K.; Oh, J.H.; Im, G.Y.; Jeong, Y.H.; Yang, M.C.; Ma, J.Y. Fermentation by Lactobacillus enhances anti-inflammatory effect of Oyaksungisan on LPS-stimulated RAW 264.7 mouse macrophage cells. BMC Complementary Altern. Med. 2012, 12, 17. [CrossRef] [PubMed]

27. Lee, J.E.; Lee, A.R.; Kim, H.; Lee, E.; Kim, T.W.; Shin, W.C.; Kim, J.H. Restoration of traditional Korean nuruk and analysis of the brewing characteristics. J. Microbiol. Biotechnol. 2017, 27, 896-908. [CrossRef] [PubMed]

28. Kim, J.E.; Jung, S.K.; Lee, S.J.; Lee, K.W.; Kim, G.W.; Lee, H.J. Nuruk extract inhibits lipopolysaccharide-induced production of nitrite and interleukin-6 in RAW 264.7 cells through blocking activation of p38 mitogen-activated protein kinase. J. Microbiol. Biotechnol. 2008, 18, 1423-1426. [PubMed]

29. Lee, D.Y.; Lee, S.J.; Kwak, H.Y.; Jung, L.; Heo, J.; Hong, S.; Kim, G.W.; Baek, N.I. Sterols isolated from Nuruk (Rhizopus oryzae KSD-815) inhibit the migration of cancer cells. J. Microbiol. Biotechnol. 2009, 19, 1328-1332. [CrossRef] [PubMed]

30. Asagiri, M.; Takayanagi, H. The molecular understanding of osteoclast differentiation. Bone 2007, 40, 251-264. [CrossRef] [PubMed]

31. Yoon, T.J.; Yoo, Y.C.; Lee, S.W.; Shin, K.S.; Choi, W.H.; Hwang, S.H.; Ha, E.S.; Jo, S.K.; Kim, S.H.; Park, W.M. Anti-metastatic activity of Acanthopanax senticosus extract and its possible immunological mechanism of action. J. Ethnopharmacol. 2004, 93, 247-253. [CrossRef] [PubMed]

32. Park, S.H.; Lee, S.G.; Kang, S.K.; Chung, S.H. Acanthopanax senticosus reverses fatty liver disease and hyperglycemia in ob/ob mice. Arch. Pharm. Res. 2006, 29, 768-776. [CrossRef] [PubMed]

33. Kimura, Y.; Sumiyoshi, M. Effects of various Eleutherococcus senticosus cortex on swimming time, natural killer activity and corticosterone level in forced swimming stressed mice. J. Ethnopharmacol. 2004, 95, 447-453. [CrossRef] [PubMed] 